\title{
Antimicrobial Susceptibility of Multidrug-Resistant Acinetobacter baumanii in a Teaching Hospital: A Two-Year Observation
}

\author{
Cucunawangsih', Veronica Wiwing1, Nata Pratama Hardjo Lugito² \\ ${ }^{1}$ Department of Microbiology, Faculty of Medicine, University of PelitaHarapan, Tangerang, Indonesia \\ ${ }^{2}$ Department of Internal Medicine, Faculty of Medicine, University of PelitaHarapan, Tangerang, Indonesia \\ Email: cucunawangsih.fk@uph.edu, veronica.wiwing@uph.edu, nata.lugito@uph.edu
}

Received 22 April 2015; accepted 2 June 2015; published 5 June 2015

Copyright (C) 2015 by authors and Scientific Research Publishing Inc.

This work is licensed under the Creative Commons Attribution International License (CC BY).

http://creativecommons.org/licenses/by/4.0/

(c) (i) Open Access

\begin{abstract}
Multidrug-resistant (MDR) Acinetobacter baumanii (A. baumanii) caused hospital acquired infection, typically in critical-ill patients with medical devices. This is a retrospective descriptive study on epidemiology and microbiology data to determine the antimicrobial susceptibility pattern of MDR-Acinetobacter baumanii isolates from a teaching hospital in Tangerang, Indonesia from Januari 2013 to December 2014. A total of 84 A. baumanii were collected. Patients suffering from respiratory tract infection had the highest number $(41.7 \%)$ of $A$. baumanii isolate. There were 39 $(46.6 \%)$ patients admitted in critical care. $A$. baumanii isolates in this study mostly were multidrug-resistant organisms with low susceptibility level to 11 antibiotic tested, $44 \%-69 \%$ in 2013 and $26 \%-67 \%$ in 2014 . A high susceptibility level was observed to amikacin $180 \%$ and $79 \%$ in 2013,2014 consecutively) and trimethoprim-sulfamethoxazole $(73 \%$ and $72 \%$ in 2013,2014 consecutively). $A$. baumanii is a hospital acquired pathogen in critically-ill patients. The susceptibility pattern of this study result showed MDR organism. There was a sharp decrease of susceptibility in all antibiotics studied from 2013 to 2014 except amikacin and trimethoprim-sulfamethoxazole.
\end{abstract}

\section{Keywords}

Multidrug-Resistant Acinetobacter baumanii, Antimicrobial Susceptibility, Amikacin

\section{Introduction}

Acinetobacter baumnaii (A. baumanii) is an opportunistic Gram-negative pathogen which has implicated in a

How to cite this paper: Cucunawangsih, Wiwing, V. and Lugito, N.P.H. (2015) Antimicrobial Susceptibility of Multidrug-Resistant Acinetobacter baumanii in a Teaching Hospital: A Two-Year Observation. Open Journal of Medical Microbiology, 5, 85-89. http://dx.doi.org/10.4236/ojmm.2015.52010 
wide range of infections, particularly in critically-ill patients with impaired immune response [1] [2]. The major characteristics of this infection include pneumonia, bacteriemia, meningitis, urinary tract infection, and surgical site infection [2] [3]. The usages of medical devices, such as vascular catheters or endotracheal tube for airway failure become the most frequent sources of Acinetobacter infections [4] [5]. In last decades, the emergence and rapid spread of Multidrug-resistant (MDR) A. baumanii causing a serious clinical problem in hospital acquired infection which is leading to an increased mortality with crude mortality rates parallel those attributed to other gram-negative bacilli (28\% - 32\%) [6] [7]. The susceptibility level of major group antibiotics used for treatment decreased rapidly and implicated in limited selection of empirical antibiotic therapy [8]. The information of these organisms and antibiotic susceptibility pattern among hospitalized patients in Indonesia is hard to find. This study was designed to determine the prevalence of MDR-A. baumanii and its antibiotic susceptibility pattern from a teaching hospital in Tangerang, Indonesia from January 2013 to December 2014.

\section{Materials and Methods}

This study was conducted in Siloam General Hospital, a new teaching hospital with 200 beds located in Tangerang, Indonesia. This was a retrospective descriptive study on epidemiology and microbiology data. The epidemiology data were collected from medical records of admitted patients with A. baumanii infection/colonization from January 2013 to December 2014. Microbiology and antimicrobial susceptibility results were extracted from laboratory data system and converted into a format which used for data analysis. The categorical data and antimicrobial susceptibility were presented as number and percentage. Identification and antibiotic susceptibility testing of all isolates was performed by an automated method from VITEX-2 Compact ${ }^{\circledR}$ (Biomérieux, France). The Interpretation of breakpoints was defined by guideline from Clinical and Laboratory Standard Institute (CLSI) [9]. Escherichia coli ATCC ${ }^{\circledR} 25922$ and Pseudomonas aeruginosa ATCC $27853^{\circledR}$ were used as control isolate for susceptibility testing.

\section{Results}

The total number of isolates was 84, consisted of 45 and 39 in 2013 and 2014 consecutively. The highest number isolate of MDR-A. baumanii infection was observed in patients suffering from respiratory tract infection who were using endotracheal tube and admitted in critical care (41.7\%).

Urinary tract infection caused by MDR-A.baumnaii (1.2\%) while blood stream infection (8.3\%). Source of MDR-A. baumanii isolate according to the specimen type was shown in Table 1 . The characteristic of A. baumanii infected patient were shown in Table 2 . The majority of patients were male (56\%) with their age ranged between 14 to 65 year-old (73.8\%). The patients with 0 to 14 years age range had the fewest number (7.2\%), followed by $>65$ years (19\%). A total of 39 (46.6\%) patients were suffering from A. baumanii infection when admitted in critical care, and there were $1(1.1 \%)$ patients admitted in pediatric department.

The frequency of MDR-A. baumanii which fluctuates during two-year observation was shown in Figure 1. The highest prevalence was in April 2013 and followed in May 2014. In accordance with this result, the number of A. baumanii isolates tested was higher in 2013 with 45 incidence and decline in 2014 with 39 incidences.

The antibiotic susceptibility level of MDR-A. baumanii to 11 antibiotic regimens was shown in Figure 2. Mostly A. baumanii isolate were multi-drug resistant. The susceptibility level was low to some antibiotic tested,

Table 1. Source of MDR-A. baumanii isolate according to the specimen type.

\begin{tabular}{ccc}
\hline Actual site of infection & Specimen type & MDR-A. baumaniin (\%) \\
\hline Respiratory tract & Sputum & $24(28.5)$ \\
& Endotracheal tube secretion & $35(41.7)$ \\
Wound & Bronchial lavage & $2(2.4)$ \\
Urinary tract & Abscess aspirate/swab & $19(22.6)$ \\
Blood stream & Urine & $1(1.2)$ \\
\hline
\end{tabular}


Table 2. General characteristic of MDR-A. baumanii from all patients.

\begin{tabular}{lccc}
\hline MDR-A. baumanii characteristic & $\mathbf{2 0 1 3}(\mathbf{n}=\mathbf{4 5})$ & $\mathbf{2 0 1 4}(\mathbf{n}=\mathbf{3 9})$ & Total (n= 84) \\
\hline Sex & $28(62.2 \%)$ & $28(71.8 \%)$ & $56(66.7 \%)$ \\
Male & $17(37.8 \%)$ & $11(28.2 \%)$ & $28(33.3 \%)$ \\
Female & & $1(2.6 \%)$ & $3(3.6 \%)$ \\
Age & $2(4.4 \%)$ & $0(0.0 \%)$ & $3(3.6 \%)$ \\
0 - 1 year & $3(6.7 \%)$ & $32(82 \%)$ & $62(73.8 \%)$ \\
1 - 14 year & $30(66.7 \%)$ & $6(15.4 \%)$ & $16(19 \%)$ \\
14 - 65 year & $10(22.2 \%)$ & & $39(46.6 \%)$ \\
$>65$ year & & $20(51.3 \%)$ & $38(45.3 \%)$ \\
Ward & $19(42.2 \%)$ & $17(43.6 \%)$ & $4(4.8 \%)$ \\
Critical care & $21(46.7 \%)$ & $0(0.0 \%)$ & $1(1.1 \%)$ \\
General & $4(8.9 \%)$ & $0(0.0 \%)$ & $2(2.2 \%)$ \\
Surgical & $1(2.2 \%)$ & $2(5.1 \%)$ & \\
Pediatric & $0(0.0 \%)$ & & \\
Maternity & & & \\
\hline
\end{tabular}

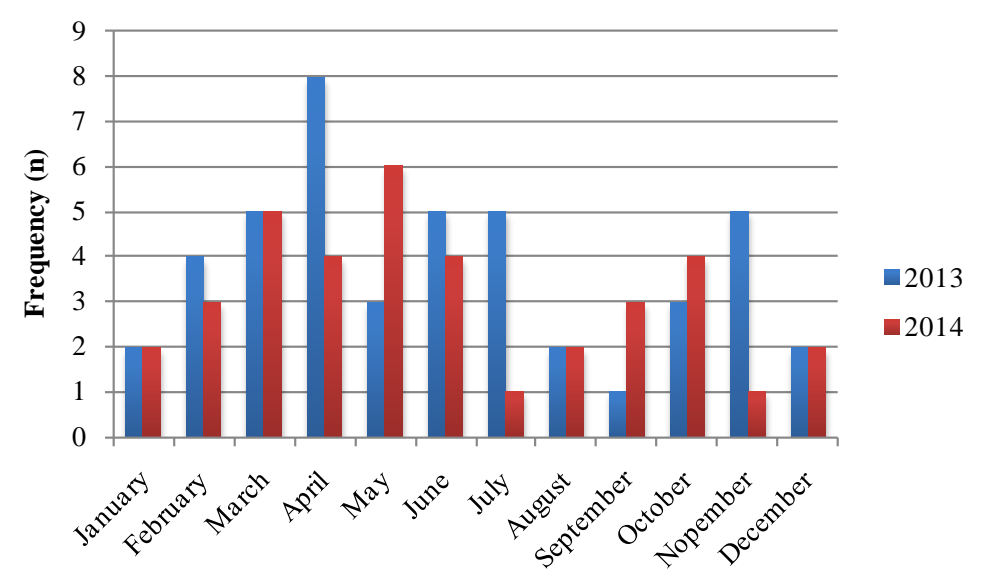

Figure 1. Frequency of MDR-A. baumanii in 2013-2014.

such as ampicillin/sulbactam69\% and 38\% in 2013 and 2014; ceftazidime 51\% and 26\% in 2013 and 2014 ; meropenem 69\% and 41\% in 2013 and 2014; levofloxacin 47\% and 33\% in 2013 and 2014. The highest susceptibility level was shown by amikacin (80\% and 80\% in 2013 and 2014) and trimethoprim-sulfamethoxazole (73\% and 72\% in 2013 and 2014). The other antibiotic also had moderate susceptibility level was tigecycline (78\% and 67\% in 2013 and 2014). In general, this study found out a sharp decreased of antibiotic susceptibility in all antibiotics studied from 2013 to 2014 except amikacin and trimethoprim-sulfamethoxazole.

\section{Discussion}

MDR-A. baumanii is a serious hospital acquired pathogen that has wide clinical spectrum, such as pneumonia, bacteriemia, urinary tract infection, surgical site infection especially in patients with medical devises, long duration of hospitalization, impaired immune response [10] [11]. Therefore, 46.6\% patients who were admitted in intensive care in this study had high risk to acquired $A$. baumanii infection. Several studies have found that patients with co-morbidities and severe ill easily infected/colonized with this organism. However, the relationship 


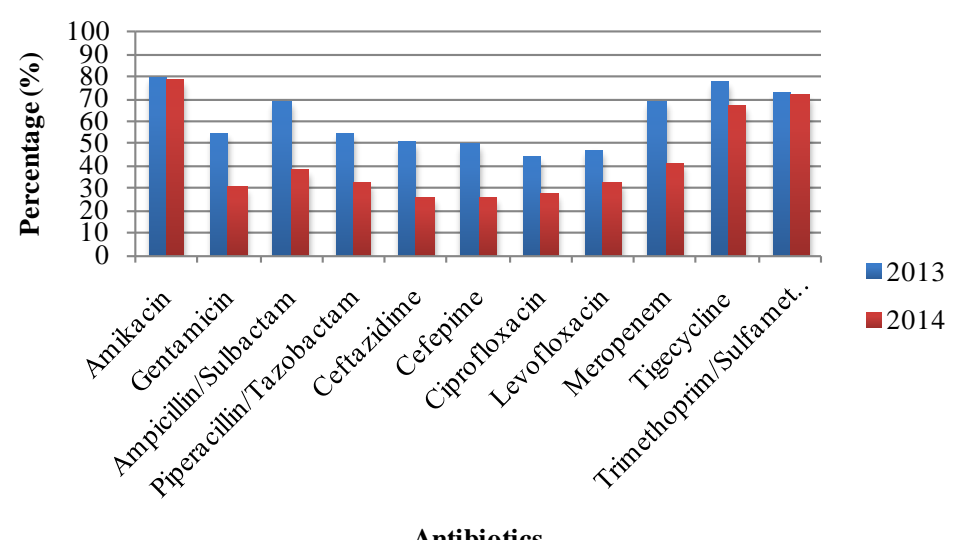

Figure 2. Changing trend of antimicrobial susceptibility in MDR-A. baumanii in 2013-2014.

between MDR-A. baumanii infection/colonization with co-morbidities did not significantly affect mortality but responsible for poor clinical outcome, need for mechanical ventilation and reduce functional status [12]. Acinetobacter baumanii can affected in any age group, from 0 year up to above 65 years, but most cases in this study were found in the aged group 14 - 65 year-old (73.8\%), followed by elderly patients 19\%. These results were similar with other study that shown MDR-A. baumanii infection was responsible for infection in patients aged group 0 - 80 year-old followed by the frequently affected in the age group above 60 years [4] [13].

A. baumanii in this study mainly isolated from lower respiratory tract (72.6\%). These findings were similar with other result where A. baumanii was recovered from $45 \%$ - 50\% patients [8] [13]. This organism also responsible for wound infection in $22.6 \%$ which much the same with the study that conducted in Saudi Arabia and Turkey, where the isolation rate was $22.3 \%$ and $27.5 \%$ [13] [14]. Bacteriemia caused by A. baumanii was found in 3.6\% isolates and much alike with the previous study [13]. The last decades, there were increase hospital acquired infections by MDR-A. baumanii globally including Indonesia [11]. All isolates in this study were resistant to almost antibiotic classes and only susceptible to amikacin and trimethoprim-sulfamethoxazole. The growing prevalence of carbapenem resistance in this study was accordance with the other study in Turkey [15] [16]. This situation is also in line with the increased of inappropriate antibiotic consumption or overuse of ciprofloxacin and carbapenems in the hospital [13] [17] [18].

\section{Conclusion}

A. baumanii is a hospital acquired pathogen in critically-ill patients. The susceptibility pattern of this study result showed MDR organism. There was a sharp decrease of susceptibility in all antibiotics studied from 2013 to 2014 except amikacin and trimethoprim-sulfamethoxazole.

\section{Conflict of Interests}

The authors declare that they have no conflict of interests regarding the publication of this paper.

\section{Acknowledgements}

The authors would like to thank Siloam Teaching Hospital in Lippo Village, Tangerang, Indonesia who made this work possible. This study was supported by Faculty of Medicine, University of PelitaHarapan University. The funders had no role in study design, analysis and interpretation of data, writing of the manuscript, and in decision to submit the manuscript for publication.

\section{References}

[1] Munoz-Price, L.S., and Weinsten, R.A. (2008) Acinetobacter Infection. New England Journal of Medicine, 358, 12711288. http://dx.doi.org/10.1056/NEJMra070741

[2] Visca, P., Seifert, H. and Towner, K.J. (2011) Acinetobacter Infection-An Emerging Threat to Human Health. 
IUBMB Life, 63, 1048-1054. http://dx.doi.org/10.1002/iub.534

[3] Wisplinghoff, H., Bischoff, T., Tallent, S.M., Seifert, H., Wenzel, R.P. and Edmond, M.B. (2004) Nosocomial Bloodstream Infections in US Hospitals: Analysis of 24, 178 Cases from a Prospective Nationwide Surveillance Study. Clinical Infectious Diseases, 39, 309-317. http://dx.doi.org/10.1086/421946

[4] Abbo, A., Carmeli, Y., Navon-Venezia, S., Seigman-Igra, Y. and Schwaber, M.J. (2007) Impact of Multidrug-Resistant Acinetobacter baumanii on Clinical Outcomes. European Journal of Clinical Microbiology and Infectious Diseases, 26, 793-800. http://dx.doi.org/10.1007/s10096-007-0371-8

[5] Cisneros, J.M. and Rodriguez-Bano, J. (2002) Nosocomial Bacteremia Due to Acinetobacter baumannii: Epidemiology, Clinical Features, and Treatment. Clinical Microbiology and Infection, 8, 687-693. http://dx.doi.org/10.1046/j.1469-0691.2002.00487.x

[6] Dijkshoorn, L., Nemec, A. and Harald, S. (2007) An Increasing Threat in Hospitals: Multidrug-Resistant Acinetobacter baumannii. Nature, 5, 939-949.

[7] Perez, F., Hujer, A.M., Hujer, K.M., Decker, B.K., Rather, P.N. and Bonomo, R.A. (2007) Global Challenge of Multidrug-Resistant Acinetobacter baumannii. Antimicrobial Agents and Chemotherapy, 51, 3471-3484. http://dx.doi.org/10.1128/AAC.01464-06

[8] Gonlugur, U., Bakici, M.Z., Akkurt, I. and Efeoglu, T. (2004) Antibiotic Susceptibility Patterns among Respiratory Isolates of Gram-Negative Bacilli in a Turkish University Hospital. BMC Microbiology, 4, 1471-2180.

[9] Clinical and Laboratory Standard Institute (2002) Performance Standards for Antimicrobial Susceptibility Testing: Twenty-Second Informational Supplement. M100-S22, 32, 64-65.

[10] Howard, A., Donoghue, M.O., Feeney, A. and Sleator, R.D. (2010) Acinetobacter baumannii: An Emerging Opportunistic Pathogen. Virulence, 3, 243-250. http://dx.doi.org/10.4161/viru.19700

[11] Dent, L.L., Marshal, D.R., Pratap, S. and Hulette, R.B. (2010) Multidrug Resistant Acinetobacter baumanii: A Descriptive Study in a City Hospital. BMC Infectious Diseases, 10, 196, 1-7.

[12] Khan, M.A., Mahomed, M.F., Ashshi, A.M. and Faiz, A. (2012) Drug Resistance Patterns of Acinetobacter baumanii in Makkah, Saudi Arabia. Pakistan Journal of Medical Research, 51, 127-131.

[13] Joshi, S.G., Litake, G.M., Satpute, M.G., Telang, N.V., Ghole V.S. and Niphadkar K.B. (2006) Clinical and Demographic Features of Infection Caused by Acinetobacter Species. Indian Journal of Medical Sciences, 60, 351-360. http://dx.doi.org/10.4103/0019-5359.27219

[14] Hsueh, P.R., Teng, L.J., Chen, C.Y., Chen, W.H., Ho, S.W. and Luh, K.T. (2002) Pandrug-Resistant Acinetobacter baumanii Causing Nosocomial Infections in a University Hospital, Taiwan. Emerging Infectious Diseases, 8, 827-832. http://dx.doi.org/10.3201/eid0805.020014

[15] Türkpğlu, M.A. and Iskit, T.A. (2008) Ventilator Associated Pneumonia Caused by High Risk Microorganisms: A Matched Case Control Study. TuberkToraks, 56, 139-149.

[16] Dizbay, M., Tunccan, O.G., Ergur-Sezer, B. and Hizel, K. (2010) Nosocomial Imipenem-Resistant Acinetobacter baumanii Infections: Epidemiology and Risk Factors. Scandinavian Journal of Infectious Diseases, 42, 741-746. http://dx.doi.org/10.3109/00365548.2010.489568

[17] Mahajan, G., Sheemar, S. and Chopra, S. (2011) Carbapenem Resistance and Phenotypic Detection of Carbapenemases in Clinical Isolates of Acinetobacter baumanii. Indian Journal of Medical Sciences, 65, 18-25. http://dx.doi.org/10.4103/0019-5359.103161

[18] Maragakis, L.L. and Perl, T.M. (2008) Acinetobacter baumannii: Epidemiology, Antimicrobial Resistance, and Treatment Options. Clinical Infectious Diseases, 46, 1254-1259. http://dx.doi.org/10.1086/529198 